\title{
GIS 多维统一计算的几何代数方法
}

袁林旺 ${ }^{(1)^{*}}$ ，问国年 ${ }^{\left(\mathbb{1}^{*}\right.}$ ，罗文 ${ }^{(1)}$, 俞肇元 ${ }^{(1)}$ ，易琳 ${ }^{(1)}$, 盛业华 ${ }^{(1)}$

(1) 南京师范大学虚拟地理环境教育部重点实验室, 南京 210046;

(2) 江苏省大规模复杂系统数值模拟重点实验室, 南京 210046

* 联系人, E-mail: yuanlinwang@njnu.edu.cn; gnlu@njnu.edu.cn

2011-08-25 收稿, 2011-10-31 接受

国家自然科学基金(41171300, 40730527, 40801149)和国家高技术研究发展计划(2009AA12Z205)资助

摘要 几何代数的多维统一与坐标无关特性为构建 GIS 多维统一计算模型奠定基础. 利用基 本几何对象的几何代数内、外积表达, 基于多重向量构建几何-拓扑结构统一的多维地理场景 对象自适应表达模型. 基于几何代数基本算子构建面向几何、拓扑以及 GIS 分析的多维运算 算子, 形成表达结构与运算结构统一, 且可支撑多维复杂场景分析的统一计算框架. 三维社 区实例验证表明, 基于几何代数的多维统一计算模型可有效支撑多维复杂地理场景表达与分 析, 并可借鉴和继承现有 GIS 算法和地理模型, 从而推进 GIS 多维统一表达、分析与建模的 发展.

\section{关键词}

几何代数

GIS

多维统一表达

多维统一计算
复杂地理对象和地理场景的组织与表达是实现 高可用性 GIS 的关键 ${ }^{[1 \sim 4]}$. 可支撑运算的对象表达与 计算模型构建, 是实现 GIS 从静态特征研究转向时 空分布结构、动态演化过程及机理模拟和预测等综合 性研究, 进而实现 GIS 与专业领域模型集成与综合 应用的重要环节 ${ }^{[58]}$. 基于欧氏几何的传统 GIS 在支 撑复杂地理对象表达、多维空间关系和地学分析上仍 存在诸多局限 ${ }^{[9]}$. 基于新的数学理论, 构建可支撑多 维地理对象统一表达与运算的表达与计算模型, 是 降低现有多维 GIS 系统架构复杂性, 提升其运算与 分析效率的可行途径 ${ }^{[10,11]}$. 几何代数作为连接代数 和几何、数学和物理、抽象时空和实体时空的统一描 述性语言, 通过“积”来定义几何、度量、拓扑等空间 操作集合，可对欧氏、齐次以及共形空间进行表达及 相互转化, 进而实现多种代数、几何系统的统一与表 达 ${ }^{[12,13]}$. 微积分、几何学及信号处理方法均可在几何 代数框架下的重构与扩展 ${ }^{[14,15]}$, 为几何代数框架下 地学模型的扩展与重构提供了借鉴. 本文基于几何 代数多重向量数学结构与几何代数算子, 构建了几 何结构与拓扑结构统一的维度自适应对象表达模型,
以及表达结构与运算结构统一的多维统一计算模型, 并以三维社区进行了实例验证.

\section{1 可支撑运算的多维统一表达}

几何代数以维度运算为基础, 利用内积、外积进 行不同维度几何对象构建, 不同维度几何对象的多 重向量统一表达可直接支撑计算. 基于几何代数的 基本运算与基本算子, 可以扩充出简洁、直观的几何 与代数运算 ${ }^{[16,17]}$, 为几何对象多维统一表达与运算 提供基础 ${ }^{[13,16]}$.

\section{1 基于片积的多维复杂对象表达}

共形几何代数 (Conformal Geometric Algebra, $\mathrm{CGA}$ ) 是最常用的几何代数系统之一 ${ }^{[18]}$, 通过引入 $e_{0}$ 和 $e_{\infty}$ 两个辅助坐标以及共形变换, 实现了不同维度 对象和不同坐标系统的统一, 并赋予内、外积明确的 几何意义. 在几何代数空间 $C l(p, q)$ 中, 任意两向量 $a, b$ 间运算规则封闭于两者的几何积(即任意运算均 可以归结至几何积运算), 两者的几何积 $a b$ 可按下式 进行展开:

英文版见: Yuan L W, Lü G N, Luo W, et al. Geometric algebra method for multidimensionally-unified GIS computation. Chin Sci Bull, 2012, 57: 802-811, doi: 10.1007/s11434-011-4891-3 
$a b=a \wedge b+a \cdot b=\langle a b\rangle_{\operatorname{grade}(a)+\operatorname{grade}(b)}+\langle a b\rangle_{\operatorname{grade}(a) \text {-grade }(b)}$.

几何积的定义表明其结果为一个混合维度的向 量, 称之为多重向量(multivector), 多重向量和片积 (blades) 是几何代数的基本数学结构, 也是支撑多维 对象统一表达和不同维度空间中空间关系解析与运 算的基础.

一个 $k$ 阶的片积是由 $k$ 个独立的向量通过外积形 成: $A_{<k>}=a_{1} \wedge a_{2} \wedge \cdots \wedge a_{k}$. 在几何代数中任意的标 量(0 阶片积)、向量、二重向量、三重向量乃至更高 维度向量均是独立的片积. 片积的几何结构定义了 一个 $k$ 维的子空间, 并使得在该 $k$ 维子空间中任意由 对象 $A$ 所确定的向量 $x$ 满足 $x \wedge \boldsymbol{A}=0^{[19]}$. 在几何结构 上, 片积可以直接表达欧氏几何空间中点、线、面等 简单几何对象，其子空间特性使其还可表达点对、几 何空间等非常规几何对象. 表 1 给出了部分基本几何 对象的内、外积表达. 相对于欧氏空间中的单形表达， 基于几何代数的几何对象均由外积(内积)直接构建, 因而同时具备了几何对象和代数表达两种特性, 可 直接用于几何计算. 如线 $L$ 相对于平面 $P$ 的投影及反 射分别为 $(\boldsymbol{L} \cdot \boldsymbol{P}) \boldsymbol{L}^{-1}$ 和 $\boldsymbol{L} \boldsymbol{P} \boldsymbol{L}^{-1}$, 即几何对象的片积表达 同时具备对象表达与运算算子的双重性质, 并在结 构和运算规则上具有一致性，从而不仅赋予了几何 代数对象表达明确的几何与代数意义，同时也为表 达结构与运算结构的统一提供了完备的数学基础.

在 CGA 中，几何对象的 Grassmann 结构与维度 结构一致，保证了基于外积的几何形体表达可反映 不同层次几何形体间的相互构建关系. 几何对象的 内积表达内蕴了对象构建的几何特性与几何关系, 度量内蕴性则可衍生出内蕴距离、角度等度量参数的 几何对象参数化表达. 而内外积表达的坐标无关性,
使得几何对象表达具有多维自适应性以及几何结构 自适应性，并可有效简化计算，如 Dorst 等人 ${ }^{[13]}$ 构建 了球面、平面自适应拟合算法. 几何形体在表达形式 及几何意义上的统一性, 可实现坐标无关且兼顾维 度构建关系和几何度量关系的基本几何对象自适应 表达，进而可同时实现基于几何层次关系、几何位置 关系以及几何度量关系的几何形体表达与建模.上 述特性为同时包含几何造型与几何关系的多维几何 对象的统一与自适应表达提供基础 ${ }^{[20 ~ 22]}$.

与 GIS 中的单纯复形结构表达类似，可以将结构 各异的多维几何对象(复合几何要素)分解成由点、线、 面、体等不同维度的单一几何要素(单一几何要素类) 的集合，并用片积对各几何要素类加以表达；应用多 重向量连接上述几何要素类的表达，可实现复杂几 何对象的几何代数表达 ${ }^{[21]}$ (图 1). 其实现层次如下: (1) 几何表达: 通过地理对象的分对象、分要素拆分, 实现不同层次地理对象的几何构造与表达，基于多 重向量进行不同维度对象统一表达与存储, 进而重 构出原始地理对象; (2) 对象组织：构建融合几何表 达与拓扑关系的多维地理对象的层次关系, 实现复 杂几何对象的层次分解, 得到不同维度上单一的几 何要素. 利用基本几何元素的几何代数表达, 将上述 结构与几何代数空间中的片积相关联，利用内积和 外积实现不同维度几何对象的相互转化与构建, 进 而基于多重向量实现不同维度片积的统一整合、表达 与存储; 3) 属性嵌人: 基于多重向量的对象表达, 建立语义描述、对象关联以及属性嵌人方法实现对象 属性嵌人. 根据原始数据的语义关联关系，对基于多 重向量表达的数据进行语义和属性配置, 并进行完 整性、一致性检验, 以保证地理对象表达的语义与属 性的一致性.

表 1 基本几何对象的内、外积表达 ${ }^{a)[18]}$

\begin{tabular}{llll}
\hline & 外积表达 & \multicolumn{2}{c}{ 内积表达 } \\
\hline 点 $A$ & $\boldsymbol{A}$ & 点 $A$ & $X \cdot \boldsymbol{A}=0$ \\
点对 $(A, B)$ & $\boldsymbol{A} \wedge \boldsymbol{B}$ & 过 $(A, B)$ 中点的平面 & $X \cdot(\boldsymbol{A}-\boldsymbol{B})=0$ \\
圆 $(A, B, C)$ & $\boldsymbol{A} \wedge \boldsymbol{B} \wedge \boldsymbol{C}$ & 过 $P$ 点方向 $\boldsymbol{A}_{k}$ 的对偶平面 & $X \cdot\left(-\boldsymbol{P} \cdot\left(\boldsymbol{A}_{k} e_{\infty}\right)\right)=0$ \\
直线 $(A, B)$ & $\boldsymbol{A} \wedge \boldsymbol{B} \wedge \boldsymbol{e}_{\infty}$ & 法向量 $\boldsymbol{n}$, 支撑向量 $\boldsymbol{\delta}$ 的对偶平面 & $X \cdot\left(\boldsymbol{n}-\boldsymbol{\delta}\|\boldsymbol{n}\| e_{\infty}\right)=0$ \\
球 $(A, B, C, D)$ & $\boldsymbol{A} \wedge \boldsymbol{B} \wedge \boldsymbol{C} \wedge \boldsymbol{D}$ & 球心 $A$ 半径 $\rho$ 的球 & $X \cdot\left(\boldsymbol{A}-0.5 \rho e_{\infty}\right)=0$ \\
平面 $(A, B, C)$ & $\boldsymbol{A} \wedge \boldsymbol{B} \wedge \boldsymbol{C} \wedge \boldsymbol{e}_{\infty}$ & 过 $P$ 点法向量为 $\boldsymbol{n}$ 的对偶平面 & $X \cdot\left(\boldsymbol{P} \cdot\left(\boldsymbol{n} \wedge \boldsymbol{e}_{\infty}\right)\right)=0$ \\
三维欧氏空间 & $\boldsymbol{A} \wedge \boldsymbol{B} \wedge \boldsymbol{C} \wedge \boldsymbol{D} \wedge \boldsymbol{E}$ & 任意几何体 $S$ 的内积表达 & $X \cdot \boldsymbol{S}=0$ \\
\hline
\end{tabular}

a) 表中黑斜体为对应对象的几何代数表达 


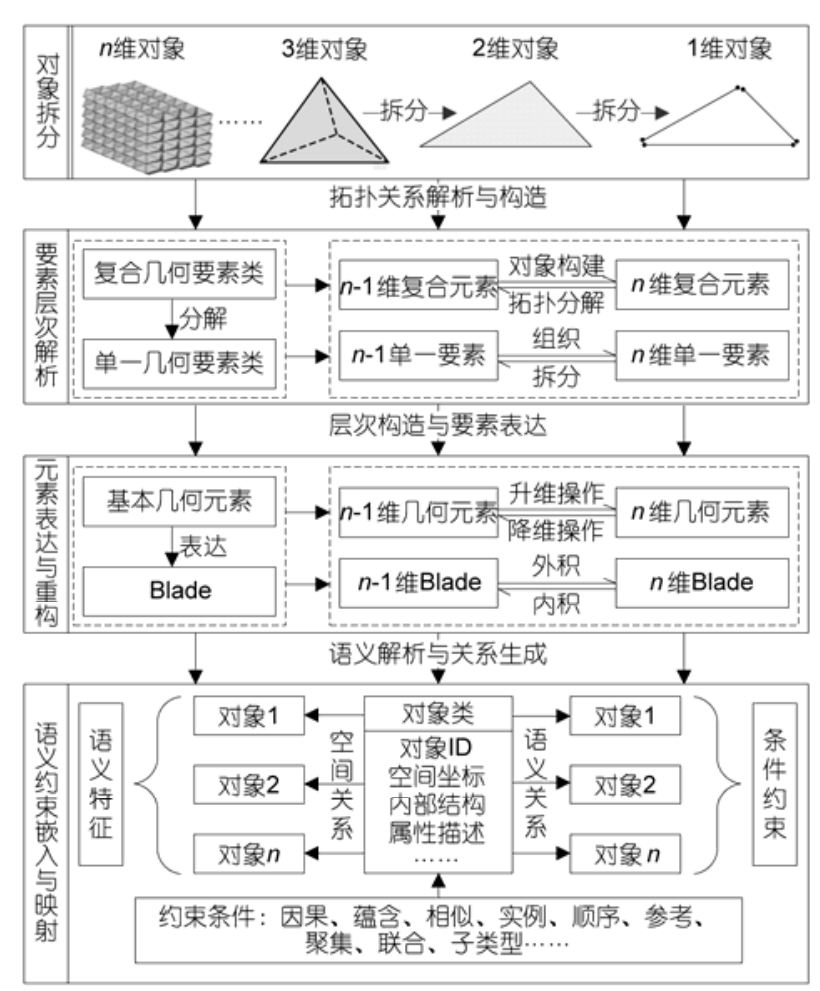

图 1 地理对象的多维统一表达

\section{2 基于多重向量的多维复杂场景统一组织}

基于片积数学结构可实现不同维度对象的一体 化表达、存储与计算. 其维度操作直接内蕴于基本代 数运算, 因而可直接利用内积和外积实现不同维度 几何对象相互转换与构建, 并可保证不同维度的对 象组织关系与其拓扑结构的一致性. 不同维度几何 形体的参数化表达, 使得地理对象结构自适应于构 成该对象的次一级几何形体变化, 降低了数据存储 量及拓扑结构与空间关系维护的难度. 而多重向量 对不同维度片积的统一表达与运算, 则为地理场景 中多维对象统一组织与存储及多维统一的几何运算 提供了运算规则与原生的数学结构. 基于多重向量 的地理场景组织的关键在于：(1) 如何基于不同的片 积及片积集合形成可进行有效数据管理及运算的多 重向量对象; (2) 如何根据运算需求从多重向量中快 速提取所需的几何对象. 图 2 展示了从简单几何元素 片积表达构建复杂地理场景多重向量表达的过程.

基本几何元素表达由包含于“[ ]”中的几何代数 表达和用于限定对象边界“< $>$ ”中的点序列两部分组 成. 由于基于片积的几何对象表达的几何维度与其
Grassmann 结构具有一致性，基本几何对象仅需与其 Grassmann 等级相对应个数的点集即可实现其所在 形体表达. 例如: 线(段)对象和多边形对象只需 2 个 点和 3 个点即可分别表达它们所在的直线 $\left(A_{1} \wedge\right.$ $\left.B_{1} \wedge e_{\infty}\right)$ 和平面 $\left(A_{1} \wedge B_{1} \wedge C_{1} \wedge e_{\infty}\right)$. 同时选择合适的 点序列按序进行边界限制, 如线段 $A_{1} B_{1}$ 和多边形 $A_{1} B_{1} C_{1} D_{1} E_{1} F_{1}$ 的限制点集分别为 $\angle A_{1}, B_{1}>$ 和 $A_{1}, B_{1}, C_{1}$, $D_{1}, E_{1}, F_{1}>$. 同理可推导出(球)体以及更高维度基本几 何元素的表达. 复合对象以及地理场景则通过 “ $\oplus$ ” 连接基本几何元素, 进而实现基于多重向量的多维 统一表达. 简单几何体“[ ]”中存储的是 Blade, 复杂 的几何对象与地理场景的“[ ]”中存储对象为多重向 量, 它们均可运用几何代数算子进行多维统一几何 与拓扑运算. 为便于场景对象管理及空间分析, 对多 重向量表达的场景对象按类型重组, 形成基于多重 向量的复杂几何对象/场景的形式化表达:

\section{GeoObjMv $=$ Obj.Points $\oplus$ Obj.Lines $\oplus$ Obj.Planes}

$$
\oplus \cdots \oplus \text { Obj.Sphere, }
$$

其中, “ $\oplus ”$ 表示不同 Blade 之间的连接符. Obj. Points, Obj.Lines 等分别为表征特定维度的片积的集合 (Bladelist). 据图 2 所示的基本几何对象的表达, 可进 一步将式(2)写成如下形式:

GeoObjMv = Obj.Points $\oplus$ Obj.Lines $<$ Lines.Pointsindex $>\oplus$, Obj.Planes $<$ Planes.Pointsindex $>\oplus \cdots \oplus$,

Obj.Sphere $<$ Sphere.Pointsindex $>$,

其中, Obj.Points, Obj.Lines 等存储的是几何对象的片 积表达，但进行外积的元素并非真正的几何对象，而 是其 ID 索引表. Lines.Pointsindex 和 Planes.Pointsindex 中存储几何对象边界限制点, 同样仅存储点的 ID 索引表. 上述索引表包含了场景中不同维度对象 间的关联关系与组织结构. 基于点集索引的多维场 景的多重向量表达，一方面可以有效表征场景中不 同维度对象的分布以及各自间组合、构建关系，同时 其形式化表达可以采用类似符号计算的方式进行规 则推理与判断, 而仅在最后需要输出数值运算(此处 的数值运算为广义的数值运算, 其结果可能是几何 对象的几何方程或表达式)结果时, 才按需进行运算, 从而可以大幅简化其计算复杂度 ${ }^{[22,23]}$.

多重向量结构中“ $\oplus$ ”仅用于连接不同维度对象, 而不进行数值计算, 通过对组成该多重向量的 Blade 结构、类型及其几何意义进行解析与提取, 可实现具 


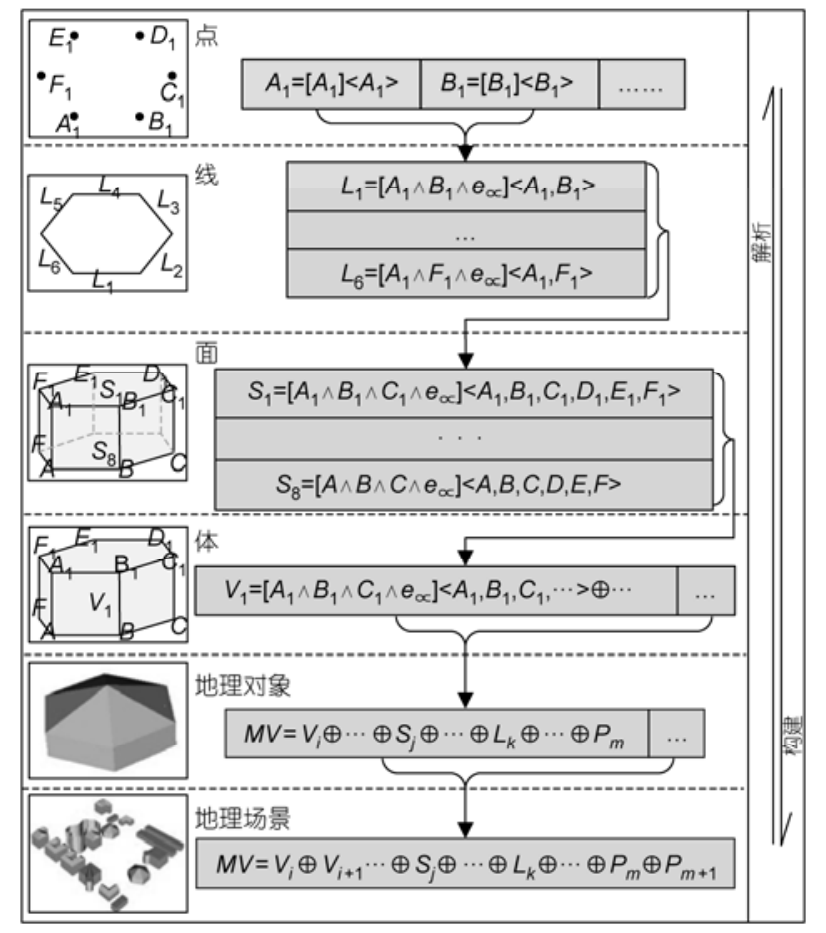

图 2 基于多重向量的地理场景组织

黑斜体为点的几何代数表达

针对性的几何表达与运算 ${ }^{[22]}$. 多重向量为多维空间 数据存储结构与存取策略设计以及结构与流程优化 提供了理论基础与实现方法. 通过运算过程中对各 Blade 的 Grade 的跟踪和判定, 不仅可解析出运算结 果中不同维度的对象及其组成, 基于不同维度对象 构成坐标基的正交性，可通过坐标约除简化计算. 基 于多重向量的地理对象表达，不仅可表征其几何构 成，且其作为一个复形集合可整体应用相关的几何 与度量算子, 获取其自身结构以及不同地理对象间 的几何与度量关系, 所构建的多数空间分析算子与 算法也可同时适用于笛卡尔坐标系和球面坐标系.

\section{2 基于几何代数的多维统一计算}

与欧氏几何基于 “形”的几何计算不同，在几何 代数框架下, 无论几何体还是几何关系均是基于几 何积的代数表达, 其表达内蕴几何特征、参数特征和 计算特征，使得同时包含对象表达与关系运算的多 维统一运算成为可能, 从而实现了存储结构、表达结 构和运算结构的统一, 进而可构造出灵活、高效的计 算空间与计算流程，简化复杂分析 ${ }^{[18,22]}$.

\section{1 表达结构与运算结构统一}

在几何代数框架下，任意几何对象的表达及其 计算均可统一至几何积, 对象表达具有明确的几何 意义, 且对象表达与运算参数直接蕴含于计算表达 式，使得其在运算过程中可自适应更新. 表达与运算 结构的统一性为基于几何代数算子的直接几何计算 提供了基础，不仅可继承原始算子所表达的几何特 性与意义, 且其结果也多具有明确的几何结构. 以求 交运算为例(图 3), 利用几何代数外积的 Grassmann 结构, 可实现不规则多面体的统一的点、线、面的层 次性表达. 应用多维统一的求交算子(meet)于此类层 次性表达，对运算过程按拓扑结构逐级分解与简化, 可充分发挥几何代数表达的关系内蕴特性, 实现表 达结构对运算结构的直接支撑.

多重向量可以实现不同维度、不同类型几何对象 的统一表达, 对其特性进行拓展后, 可实现对复杂地 理对象与空间关系的一体化、形式化表达. 在几何代 数框架下, 多数的几何、拓扑关系算子及其运算结果 也可表达成多重向量，从而可设计同时包含几何对 象与关系集合的新型存储结构. 相对于静态对象关 系存储方法, 该方案具有更好的自适应性与动态性, 其关系可参数化表达, 且可与几何对象同时应用相 关算子进行分析. 基于几何代数不仅可建立多维统 一描述框架，还可有效地借鉴和继承现有的算法和 模型, 从而支撑地理现象和地理对象的多维表达、分 析与建模.

\section{2 多维统一计算的自适应性}

多维统一运算是 GIS 支撑地理模型分析的关

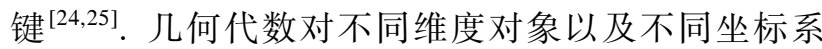
统的统一, 为几何对象的多维统一表达与运算提供 基础. 几何代数的坐标无关性与维度无关性使其表 达的几何特征是几何对象自身内蕴的几何特征，计 算获得的几何关系也是各几何对象间不依赖坐标的 相对关系，从而可用于几何对象的自适应表达、动态 更新与关系计算. 图 4 所示为两几何对象的求交流程, meet 算子计算结果自适应于求交对象，仅与对象自 身的几何特性有关，而与其所在维度、所处坐标以及 表达形式无关，进而可构建几何代数框架下的统一 计算流程。

基于多重向量的表达结构使得几何对象及其关 系表达在满足多维统一性的同时，又具有较好的独 


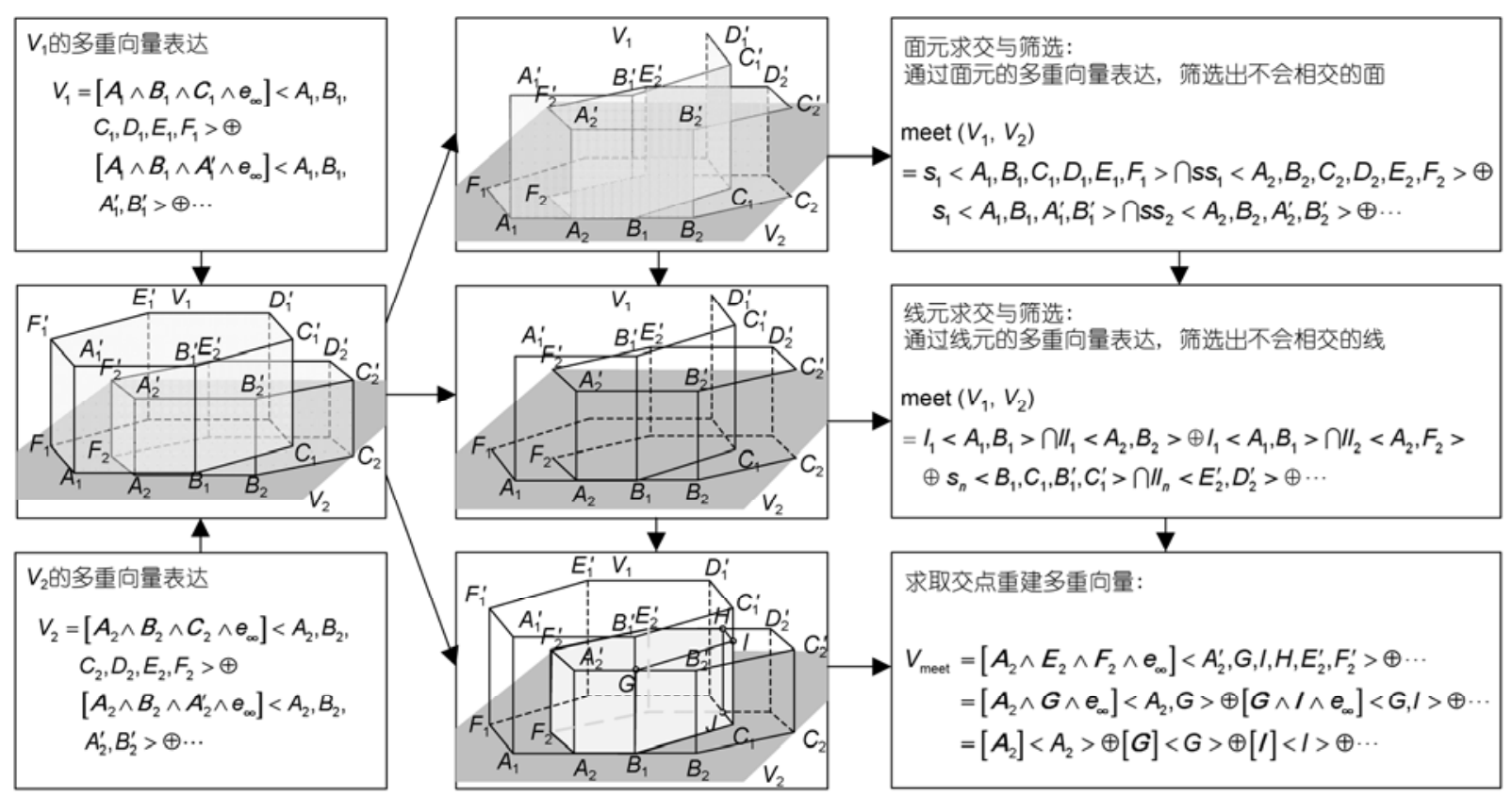

图 3 表达结构与计算结构的统一

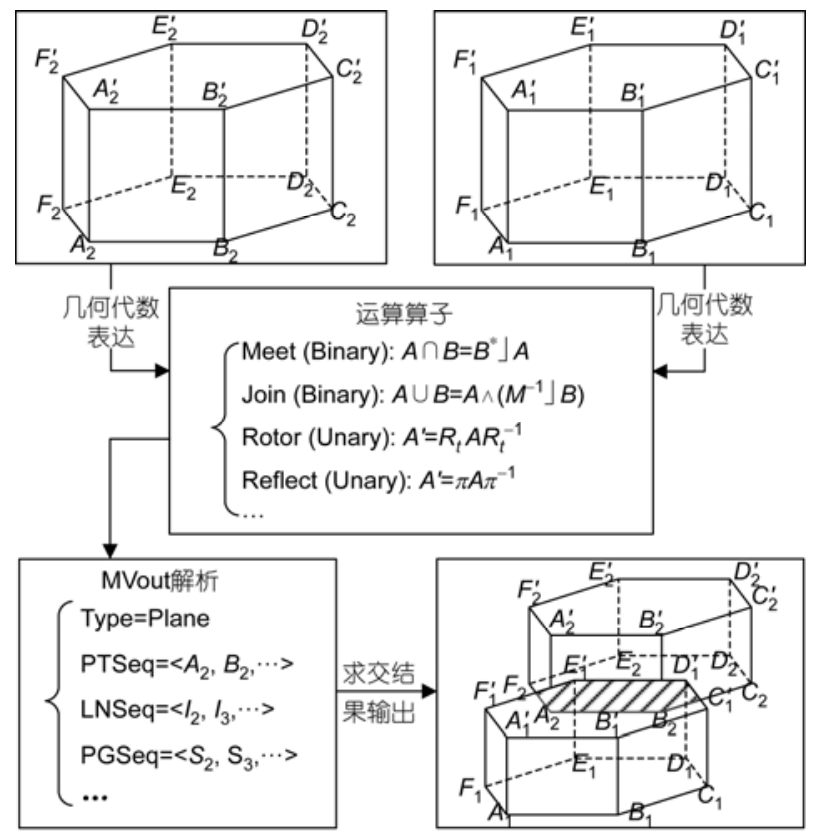

图 4 多维几何对象的自适应计算

立性; 表达的形式化、参数化与维度的自适应性特征 保障了算法的简洁性与通用性 ${ }^{[12,26]}$. 通过几何代数 算子实现的多维 GIS 计算具有多维统一性、对象自适 应性以及运算独立性等特点, 且基于算子的运算结 果本身也是一个具有明确几何意义的多重向量, 因 此可以构建多重向量对象及关系解析算法, 实现对
多重向量的解析和计算结果的输出.

\section{3 面向 GIS 分析的多维统一计算流程与算子集}

GIS 算子的 CGA 表达几何意义明确, 可支撑 GIS 多维统一计算. 如变换算子构成组分的结构特征解 析, 可直接计算移动方向、角度、旋转轴等状态参 数 ${ }^{[22]}$. 基本算子与变换算子运算具有可叠加、整体结 构保形和顺序无关等特性，通过算子组合可实现复 杂的分析功能, 并可通过算子运算顺序调整实现算 法优化 ${ }^{[23,27]}$. 基于 CGA 还可实现对运动对象的统一 表达，如可以基于 Versor 构建同时包含平移、旋转和 缩放变换的三维几何对象复合运动的统一表达与插 值算法 ${ }^{[20,28]}$. 表 2 给出了部分常用算子的几何代数定 义及其几何意义, 基于几何代数算子的几何度量、变 换以及空间关系计算多直接内蕴了几何对象的形式 化表达且几何意义明确, 可实现表达结构与运算结 构的有效统一，进而可发展空间分析算法 ${ }^{[18,22]}$.

基于多重向量的对象表达与场景组织可直接支 撑计算, 进而可利用多维统一的几何代数算子实现 算法构建与 GIS 计算. 基于几何对象多维统一的几 何代数表达，通过定义上述几何代数算子与运算框 架, 辅以可同时支撑几何代数系统构建、多维向量表 达运算以及多对象存储容器的数据对象与数据结构, 可构建多维 GIS 空间分析的统一框架. 通过对相关 
表 2 多维统一计算算子集 ${ }^{\text {a) }}$

\begin{tabular}{|c|c|c|c|}
\hline 类型 & 算子名称 & 几何代数表达 & 描述 \\
\hline \multirow[t]{3}{*}{ 基本算子 } & 投影 & $P=(\boldsymbol{A} \cdot \boldsymbol{B}) \boldsymbol{B}^{-1}$ & $A$ 在 $B$ 上的投影 \\
\hline & 反射 & $\left\{\begin{array}{l}R=M x M^{-1} \\
R=-M x M^{-1}\end{array}\right.$ & 对象 $x$ 在 $M$ 上的反射 \\
\hline & 对偶 & $A^{*}=A I^{-1}$ & 广义法子空间求解关系化简与计算 \\
\hline \multirow[t]{3}{*}{ 变换算子 } & 缩放 & $I\left(x_{e}\right)=\frac{\rho^{2}\left(x_{e}-c_{e}\right)}{\left(x_{e}-c_{e}\right)^{2}}+c_{e}$ & 基于球或圆的缩放 \\
\hline & 平移 & $T_{a}=1+\frac{1}{2} a e_{\infty}=e^{-\frac{a}{2} e_{\infty}}$ & 沿向量 $a$ 平移 \\
\hline & 旋转 & $R_{\theta}=\cos \left(\frac{\theta}{2}\right)-\sin \left(\frac{\theta}{2}\right) \boldsymbol{L}=e^{\frac{\theta}{2} L}$ & 以 $L$ 为转轴, $\theta$ 为旋转角度的旋转 \\
\hline \multirow[t]{6}{*}{ 度量关系算子 } & 点点距离 & $d^{2}(A, B)=-2 \boldsymbol{A} \cdot \boldsymbol{B}$ & 点 $A$ 到点 $B$ 距离的平方 \\
\hline & 点线距离 & $d(A, L)=\left(e_{\infty} \wedge \boldsymbol{A} \wedge \boldsymbol{L} \wedge \boldsymbol{B}\right)$ & 带符号的线 $L$ 到点 $A$ 的距离 \\
\hline & 占圆关系 & $\underline{A \wedge B} \wedge \boldsymbol{C} \wedge \boldsymbol{D}$ & 点 $D$ 与圆 $A B C$ 之间的关系 \\
\hline & & $\overline{e_{\infty} \wedge \boldsymbol{A} \wedge \boldsymbol{B} \wedge \boldsymbol{C}}$ & 为正则点在圆内, 为负则点在圆外 \\
\hline & 圆圆关系 & $((\boldsymbol{A} \wedge \boldsymbol{B} \wedge \boldsymbol{C}) \cap(\boldsymbol{D} \wedge \boldsymbol{E} \wedge \boldsymbol{F}))^{2}$ & $\begin{array}{l}\text { 圆 } A B C \text { 与圆 } D E F \text { 之间的关系 } \\
\text { 为正两圆相交/为零两圆相切/为负两圆相离 }\end{array}$ \\
\hline & 线圆关系 & $\left(\left(e_{\infty} \wedge \boldsymbol{A} \wedge \boldsymbol{B}\right) \cap(\boldsymbol{C} \wedge \boldsymbol{D} \wedge \boldsymbol{E})\right)^{2}$ & $\begin{array}{l}\text { 线 } A B \text { 与圆 } C D E \text { 之间的关系 } \\
\text { 为正线圆相交/为零线圆相切/为负线圆相离 }\end{array}$ \\
\hline \multirow[t]{4}{*}{ 拓扑关系算子 } & 左缩进 & $A\rfloor B=A \cdot B$ & 求取 $A$ 中与 $B$ 差别最大的部分 \\
\hline & 右缩进 & $A\lfloor B=B \cdot A$ & 求取 $B$ 中与 $A$ 差别最大的部分 \\
\hline & Meet & $A \cap B=B^{*} \cdot A$ & 求取 $A$ 与 $B$ 的交 \\
\hline & Join & $\left.\boldsymbol{A} \cup \boldsymbol{B}=\boldsymbol{A} \wedge\left(M^{-1}\right\rfloor \boldsymbol{B}\right)$ & 求取 $A$ 与 $B$ 的并 \\
\hline
\end{tabular}

a) 表中黑斜体为对应对象的几何代数表达

功能函数及算子算法的定义, 实现数据输人、对象分 析、几何代数运算、运算结果解析以及数据输出及可 视化的全过程. 其核心在于通过函数定义, 从地理对 象数据中提取、解析并转换成共形空间中可进行几何 运算的数据类型与数据结构. 利用相关分析算子与 GIS 分析算法进行问题求解与计算. 几何对象表达结 构上的一致性以及几何代数运算的多维统一性、坐标 无关性和特征内蕴性, 使得多数几何代数算子均可 自适应于不同维度、不同类型的对象, 且其结果仍为 几何意义明确的多重向量表达.

\section{3 多维统一表达与计算应用示范}

\section{1 多维统一表达与计算模型构建思路}

以几何代数理论为基础，应用片积和多重向量 对多维几何对象统一表达及运算的直接支撑, 构建多 维对象的拆分与场景重构机制, 实现基于多重向量的 多维复杂地理场景的统一表达、存储与计算(图 5). 在 对象表达层面, 通过多源场景数据解析进而运用内

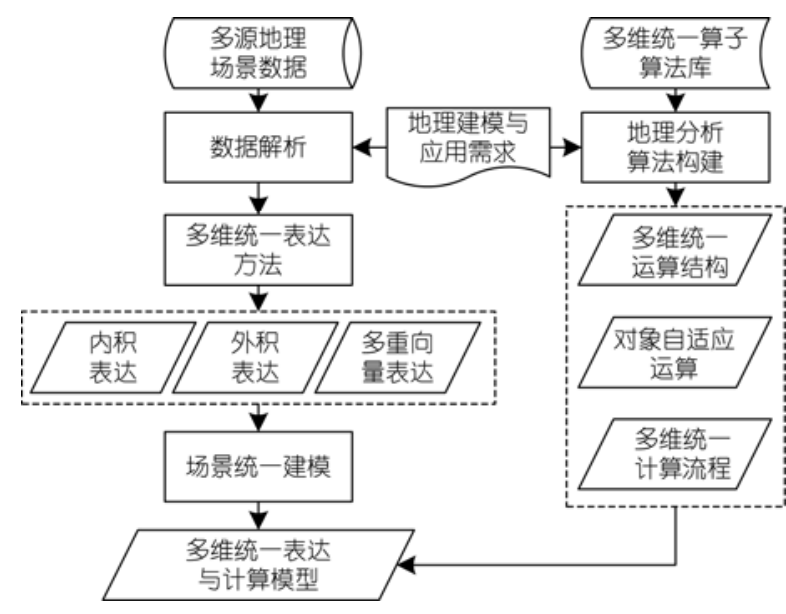

图 5 GIS 多维统一计算模型构建思路

积表达、外积表达与多重向量表达等多维统一表达方 法实现场景的统一建模; 在运算层面，基于几何代数 理论结合地理分析需求构造运算空间, 实现 GIS 空 间数据向几何代数空间的空间转换机制与关系映射 方法，进而面向几何、拓扑关系以及更复杂的空间分 
析需求构造相应的运算规则及算子算法. 基于多重 向量表达的多维场景运算具有多维统一的运算结构、 对象自适应的运算方法和多维统一的计算流程, 从 而可为多维场景的统一计算提供支持.

\section{2 面向三维社区应用示范}

基于上述多维统一表达与计算模型框架, 在基 于 Clifford 代数的时空统一分析系统(CAUSTA) $)^{[18]}$ 中, 对上述表达方式及计算方法进行实现, 并以德国 Waldbruecke 村的三维社区的建模、空间分析及应用 为例进行案例分析, 重点演示了多维复杂场景中的
对象建模与场景组织、多维场景中几何、拓扑关系的 统一计算, 进而面向地学分析构建空间插值与网络 分析算法, 并以污染物扩散条件下的应急疏散应用 为例对上述算法进行验证. 上述建模与计算过程均 在几何代数框架下实现, 并以多重向量的形式予以 表达，可直接支撑各类后续的空间分析.

首先对 CityGML 格式的社区建筑数据、道路网 络等数据进行解析, 应用基于几何代数的多维统一 数据模型和索引机制 ${ }^{[23,28]}$, 进行几何对象的空间转 换和对象表达、编码，构建多重向量场景(图 6(a)); 综 合利用 Grade 等算子和对象索引表构建基于几何代

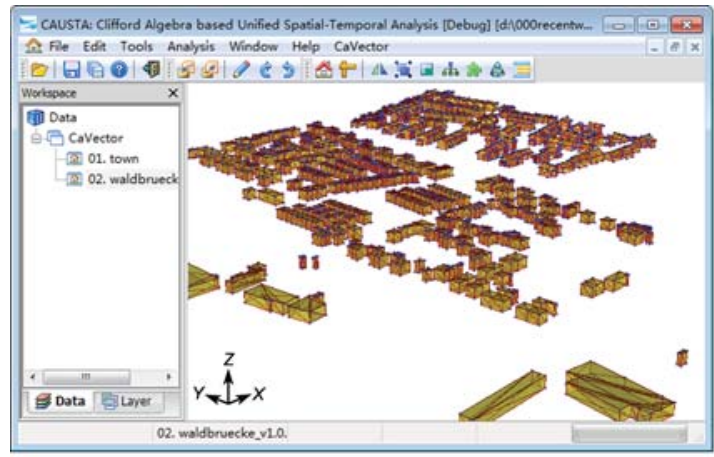

(a)

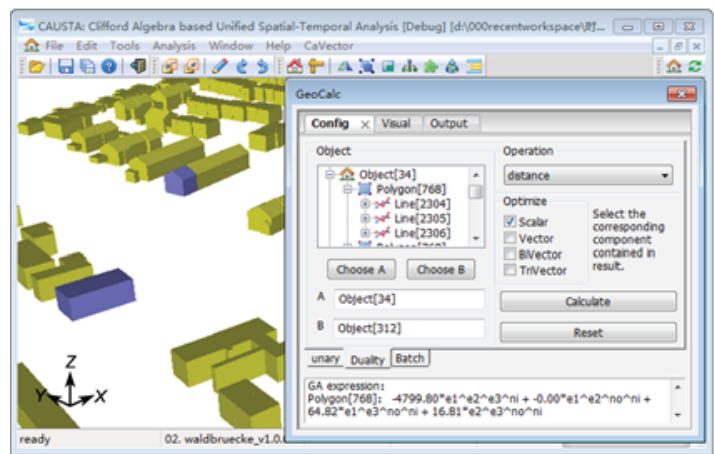

(c)

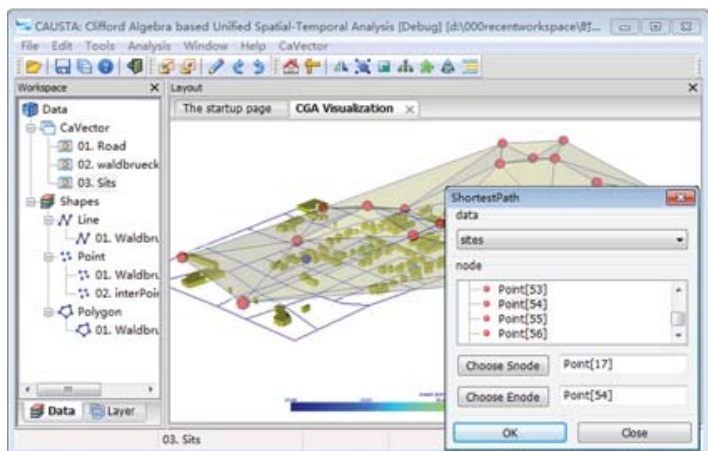

(e)

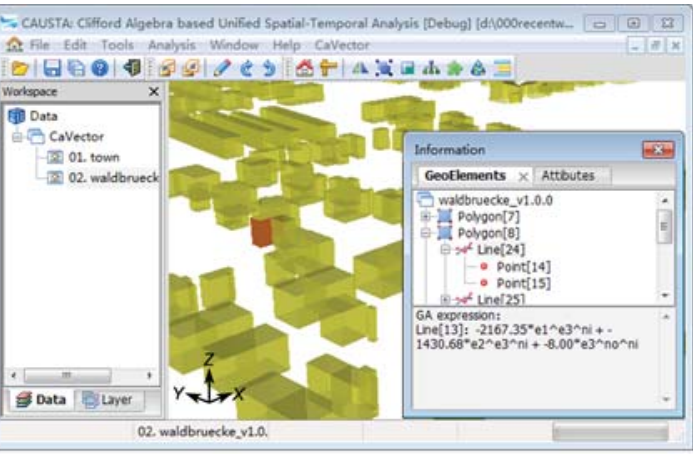

(b)

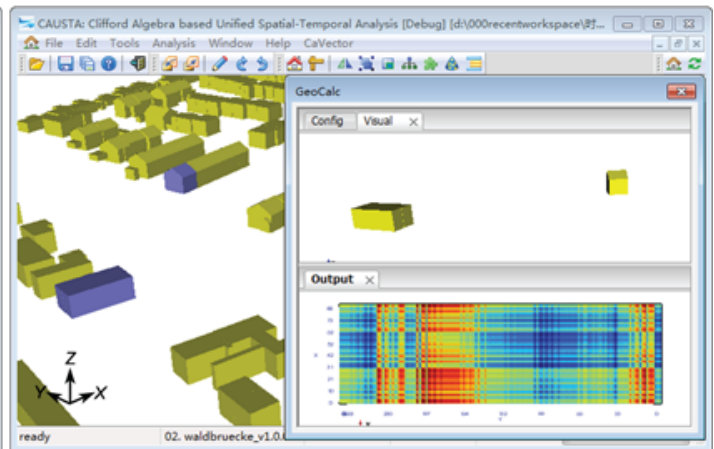

(d)

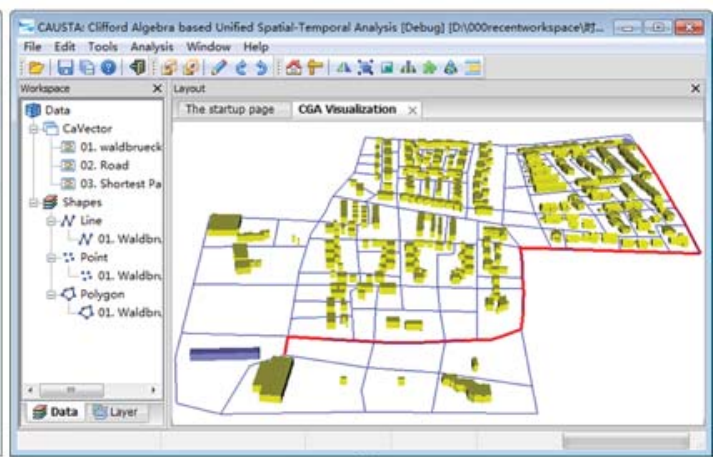

(f)

图 6 基于几何代数的多维统一分析系统分析实例

(a) 场景数据建模; (b) 场景对象查询; (c) 几何计算参数设置; (d) 几何计算结果显示; (e) 网络分析数据导人; (f) 网络分析计算结果 
数表达的场景对象查询与检索方法，实现对不同维 度对象及其几何代数表达的检索与互操作(图 6(b)); 通过对多重向量表达的各几何对象间几何计算参数 的设置, 利用几何代数算子实现基本的几何、拓扑运 算(图 6(c)); 利用几何代数算子运算的独立性构建对 象间距离、拓扑关系的同步计算算法, 实现对象间几 何拓扑关系的批量计算(图 6(d)); 面向地学应用将三 维场景、污染物采样点数据以及道路网络进行叠加; 在基于共形几何代数的多维统一 Voronoi 算法和最 短路径分析算法 ${ }^{[27,29]}$ 等基础上，建立污染物空间离 散化模型(图 6(e)), 实现污染物浓度约束下的应急疏 散路线计算(图 6(f)).

\section{4 结束语}

三维以及高维对象的表达、建模与分析是未来 GIS 发展的方向 ${ }^{[30 ~ 35]}$. 本文引人几何代数理论, 基于
基本几何对象的内、外积表达，构建了兼顾几何结构 与拓扑结构、不同维度对象统一的几何对象表达机制, 实现了具有维度自适应与几何结构自适应的多维几 何对象统一表达, 进而实现了兼顾对象维度层次关 系与几何度量关系, 基于多重向量的多维动态场景 的统一组织与表达. 构建了基于几何代数的多维统 一计算框架, 实现了表达结构、运算结构以及分析结 构的统一, 并可有效支撑复杂多维场景的统一表达 和运算. 三维社区案例分析显示, 基于几何代数的多 维统一表达与计算模型可以有效支撑复杂地理场景 的建模、运算与分析. 因此, 基于几何代数构建多维 统一数据模型, 对多维地理场景进行一体化表达与 建模, 并进行 GIS 分析算法实现以支持地理分析, 可 望形成从理论架构 - 数据模型 - 数据表达一数据分 析有机融合的基本框架与应用平台, 为地理现象发 展演化过程表达、建模与模拟提供新的技术支撑.

\section{参考文献}

1 Longley P A, Goodchild M F, Maguire D J, et al. Geographic Information Systems and Science. 2nd ed. New York: Wiley, 2005. 61-83

2 Lan H X, Martin C D, Lim C H. RockFall analyst: A GIS extension for three-dimensional and spatially distributed rockfall hazard modeling. Comput Geosci, 2007, 33: 262-279

3 Beni L H, Mostafavi M A, Pouliot J, et al. Toward 3D spatial dynamic field simulation within GIS using kinetic voronoi diagram and delaunay tetrahedralization. Int J Geogr Inf Sci, 2011, 25: 25-50

4 Castrillon M, Jorge P A, Lopez I J, et al. Forecasting and visualization of wildfires in a 3D geographical information system. Comput Geosci, 2011, 37: 390-396

5 Xie M W, Zhou G Y, Tetsuro E. Gis component based 3d landslide hazard assessment system: 3dslopegis. Chin Geogr Sci, 2003, 13: 66-72

6 Merwade V, Cook A, Coonrod J. GIS techniques for creating river terrain models for hydrodynamic modeling and flood inundation mapping. Environ Modell Softw, 2008, 23: 1300-1311

7 Naoum S, Tsanis I K, Fullarton M A. GIS pre-processor for pollutant transport modeling. Environ Modell Softw, 2005, 20: 55-68

8 Karssenberg D, De Jong K. Dynamic environmental modelling in GIS: 1. Modelling in three spatial dimensions. Int J Geogr Inf Sci, 2005, 19: 559-579

9 McIntosh J, Yuan M. A framework to enhance semantic flexibility for analysis of distributed phenomena. Int J Geogr Inf Sci, 2005, 19: 999-1018

10 Goodchild M F, Yuan M, Cova T J. Towards a general theory of geographic representation in GIS. Int J Geogr Inf Sci, 2007, 21: 239-260

11 Liu Y, Goodchild M F, Guo Q, et al. Towards a general field model and its order in GIS. Int J Geogr Inf Sci, 2008, 22: 623-643

12 Doran C, Lasenby A. Geometric Algebra for Physicists. Cambridge: Cambridge University Press, 2003. 228-264

13 Dorst L, Fongijne D, Mann S. Geometric Algebra for Computer Science: An Object-oriented Approach to Geometry. San Fransisco: Morgan Kaufmann, 2007. 355-379

14 Hestenes D, Sobcyk G. Clifford Algebra to Geometric Calculus. Heidelberg: Springer-Verlag, 1984

15 Hildenbrand D. Geometric computing in computer graphics using conformal geometric algebra. Comput Graph-UK, 2005, 29: 795-803

16 Perwass C. Geometric Algebra with Applications in Engineering. Heidelberg: Springer-Verlag, 2009. 255-368

17 Bayro-Corrochano E J, Reyes-Lozano L H, Zamora-Esquivel J. Conformal geometric algebra for robotic vision. J Math Imaging Vis, 2006, 24: $55-81$

18 Yuan L W, Yu Z Y, Chen S F, et al. CAUSTA: Clifford algebra based unified spatio-temporal analysis. Trans GIS, 2010, 14(Suppl): 59-83 
19 曹文明, 冯浩. 仿生模式识别与信号处理的几何代数方法. 北京: 科学出版社, 2010.35-36

20 李洪波. 共形几何代数与运动和形状的刻画. 计算机辅助设计与图形学学报, 2006, 18: 895-901

21 Karimipour F, Delavar M, Frank A U. A simplex-based approach to implement dimension independent spatial analyses. Comput Geosci, 2010, 36: 1123-1134

22 袁林旺, 俞肇元, 罗文, 等. 基于共形几何代数的 GIS 三维空间数据模型. 中国科学：地球科学, 2010, 40: 1740-1751

23 俞肇元. 基于几何代数的多维统一 GIS 数据模型研究. 博士学位论文. 南京: 南京师范大学, 2011

24 王生生, 刘杰, 王新颖, 等. 混合维拓扑和尺寸关系的定性空间推理. 计算机研究与发展, 2009, 46: 1169-1175

25 问国年. 地理分析导向的虚拟地理环境: 框架、结构与功能. 中国科学：地球科学, 2011, 41: 549-561

26 Friedman Y, Gofman Y. Why does the geometric product simplify the equations of physics? Int J Theor Phys, 2002, 41: 1841-1855

27 张季一. 基于 Clifford 代数的 GIS 网络分析算法研究. 硕士学位论文. 南京: 南京师范大学, 2010. 1-53

28 罗文. 基于几何代数的时空场数据特征分析与运动表达. 硕士学位论文. 南京: 南京师范大学, 2011. 1-59

29 易琳. 基于共形几何代数的多维统一 Voronoi 算法及其应用研究. 硕士学位论文. 南京: 南京师范大学, 2011. 1-50

30 杨必胜, 李清泉, 龚健雅. 一种快速生成和传输多分辨率三维模型的稳健算法. 科学通报, 2006, 51: 1589-1594

31 李清泉, 李德仁. 三维空间数据模型集成的概念框架研究. 测绘学报, 1998, 27: 325-330

32 方裕，周成虎，景贵飞，等. 第四代 GIS 软件研究. 中国图象图形学报, 2001, 6A: 817-823

33 陈军, 李志林, 蒋捷, 等. 多维动态 GIS 空间数据模型与方法的研究. 武汉大学学报(信息科学版), 2004, 29: 858-862

34 吴立新, 陈学习, 车德福, 等. 一种基于 GTP 的地下真 3D 集成表达的实体模型. 武汉大学学报(信息科学版), 2007, 32: 331-335

35 胡庆武, 林春峰, 余飞, 等. 多维 GIS 矿产评价数据管理系统设计和实现. 地球科学进展, 2010, 25: 990-995 